\title{
Probing atomic-scale symmetry breaking by rotationally invariant machine learning of 4D-STEM Data.
}

\author{
Mark Oxley ${ }^{1}$, Maxim Ziatdinov ${ }^{2}$, Ondrej Dyck ${ }^{3}$, Andrew R. Lupini ${ }^{3}$, Rama Vasudevan ${ }^{3}$ and Sergei Kalinin ${ }^{1}$ \\ ${ }^{1}$ Center for Nanophase Materials Sciences, Oak Ridge National Laboratory, Oak Ridge, Tennessee, United \\ States, ${ }^{2}$ Computational Sciences and Engineering Division, Oak Ridge National Laboratory, Oak Ridge, \\ Tennessee, United States, ${ }^{3}$ Oak Ridge National Laboratory, United States
}

The development of high-speed electron detectors has led to rapid advances in the field of scanning nano diffraction often referred to as 4D-STEM. Most analysis relies on the determination of the centre of mass $(\mathrm{COM})$ of the convergent beam electron diffraction (CBED) pattern. Such measurements have been used to determine the local electric fields [1] or the scattering potential [2]. There is limited applicability of linear separations methods such as principal component analysis (PCA) to 4D-STEM data. Such methods will label CBED patterns that are nominally the same, up to a rotation, as separate components. In this talk we will discuss the implementation of an approach for the systematic exploration of 4D-STEM data sets using rotationally invariant variational autoencoders ( $\mathrm{rVAE}$ ), which is designed to disentangle the general rotation of the object from other latent representations.

In Fig. 1 we illustrate the application of this method to simulated 4D-STEM data for $60 \mathrm{kV}$ electrons incident on graphene. Here we have used two latent spaces as well as the rotation, so this is referred to as $3 \mathrm{D}$ rrVAE. The structure used in the simulations is shown in Fig. 1 (b) and the orientation and magnitude of the calculated COM shift are shown in Fig. 1 (c) and (d) respectively. The latent space shown in Fig. 1 (a) has little variation along the vertical axis but has a significant contrast change along the horizontal axis. The rotation map obtained by rrVAE, shown in Fig. 1 (e) is similar to the calculated rotation map but opposite in direction. The first latent space distribution, shown in Fig. 1 (f) is clearly defined and exhibits the reverse contrast to the magnitude of the COM. We use the Pearson $r$ factor as a measure of the correlation between the COM magnitude and the first latent space. A value of -0.9917 shows a strong negative correlation. The second latent space distribution in bears little resemblance to the COM magnitude and has a much lower magnitude.

In Fig. 2 the results of applying the 3D rrVAE to experimental 4D-STEM data from graphene are shown. The simultaneously acquired ADF image, shown in Fig. 2 (b) clearly shows the graphene lattice. The orientation and magnitude of the calculated COM shift are shown in Fig. 2 (c) and (d) respectively. There is very little variation in the latent space shown in Fig 2 (a). The orientation map obtained by rrVAE, shown in Fig 2 (e) is in good agreement with the orientation map calculated from the COM. The second latent space distribution in Fig. 2 (g) has a reasonable negative correlation to the COM magnitude. The other latent space exhibits the periodicity of the COM magnitude but has a low correlation and is difficult to interpret.

In this presentation we will discuss the use of rrVAE on 4D-STEM data from graphene with symmetry breaking impurities and defects using a varying number of latent spaces. We will also discuss recent developments in variational autoencoders and their application to 4D-STEM simulations and experiment. 

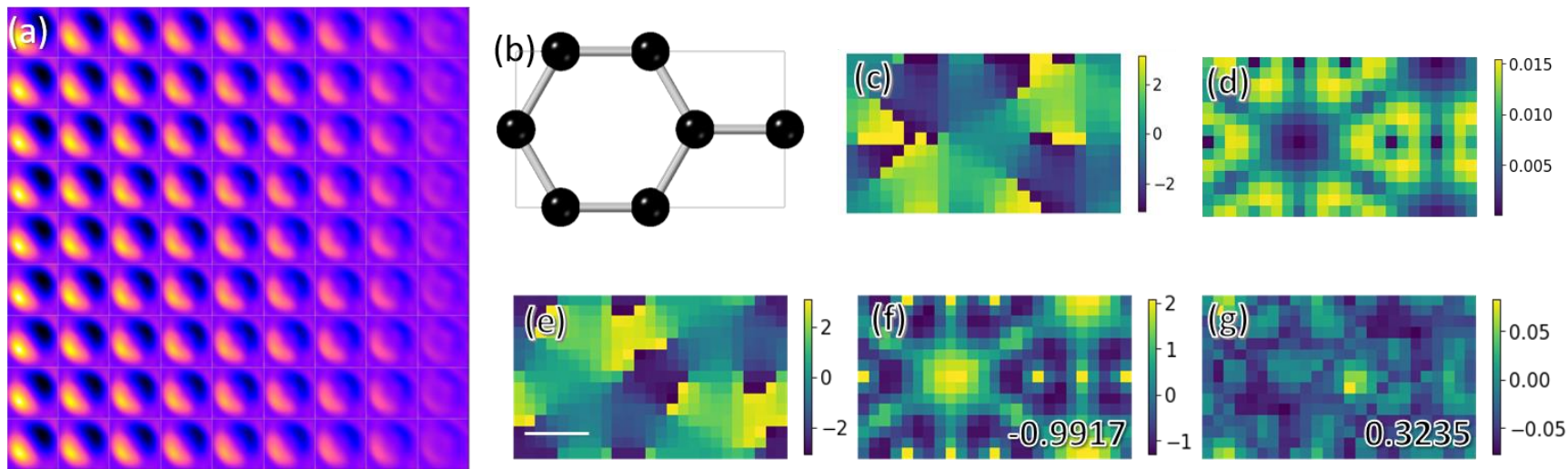

Figure 1. (a) Latent space for 3D rrVAE of simulated graphene CBED patterns for microscope operating a 60 $\mathrm{kV}$ with a $31 \mathrm{mrad}$ probe forming aperture. (b) Model of the unit cell used for multi-slice simulation. (c) and (d) angle and magnitude of the COM deflection calculated from the simulated CBED patterns, respectively. Scale bar on (e) is one $\AA$ (e) Rotation map obtained from rrVAE analysis. (f) and (g) show two latent space distributions (with Pearson $r$ factor inset).
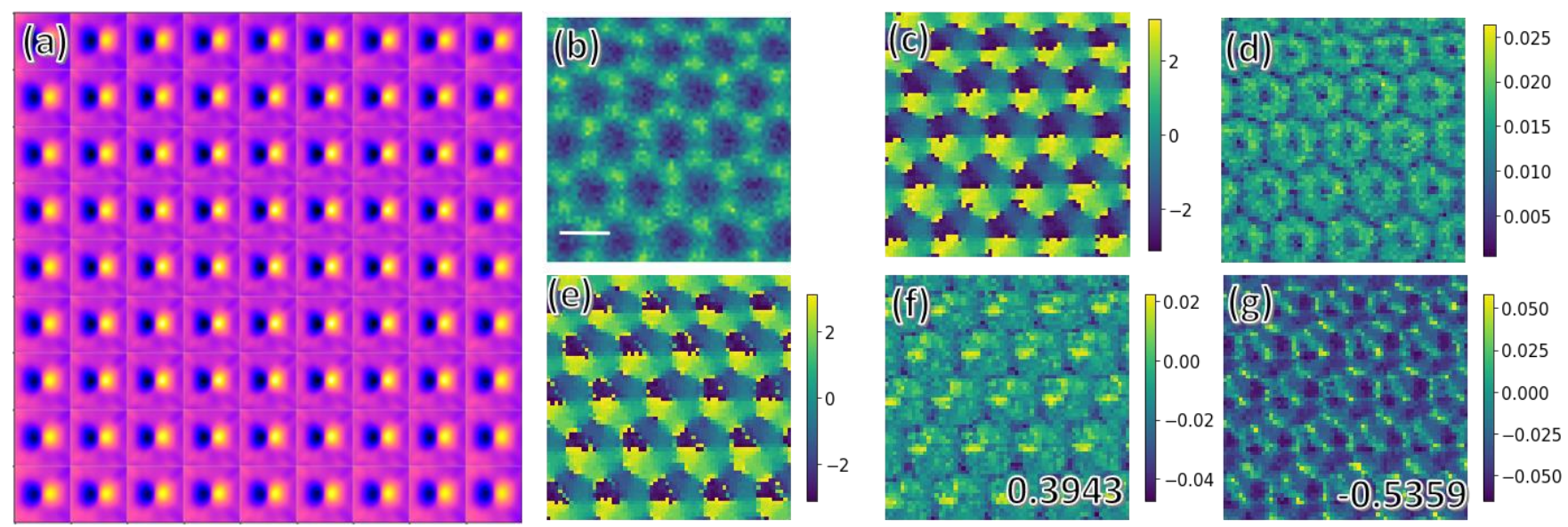

Figure 2. (a) Latent space for 3D rrVAE for experimental graphene CBED patterns with the microscope operating a $60 \mathrm{kV}$ with a $31 \mathrm{mrad}$ probe forming aperture. (b) Simultaneously acquired ADF-STEM image. (c) and (d) angle and magnitude of the COM deflection calculated from the experimental CBED patterns, respectively. (e) Rotation map obtained from rrVAE analysis. (f) and (g) show two latent space distributions with Pearson $r$ factor inset. 75 iterations were used. The scale bar in (b) is two $\AA$.

\section{References}

[1] Müller, K. et al. Nature communications 5 (2014), p. 1.

[2] Close, R. et al., Ultramicroscopy 159 (2015), p. 124.

[3] This effort (ML and STEM) is based upon work supported by the U.S. Department of Energy (DOE), Office of Science, Basic Energy Sciences (BES), Materials Sciences and Engineering Division (M.P.O., A.R.L., S.V.K., O.D.) and was performed and partially supported (M.Z.) at the Oak Ridge National Laboratory's Center for Nanophase Materials Sciences (CNMS), a U.S. Department of Energy, Office of Science User Facility. This research used resources of the Compute and Data Environment for Science (CADES) at the Oak Ridge National Laboratory, which is supported by the Office of Science of the U.S. Department of Energy under Contract No. DE-AC05-00OR22725. 